\title{
La generalización del entrenamiento en las técnicas de evaluación del potencial de aprendizaje
}

\author{
MARÍA DOLORES CALERO \\ Universidad de Granada \\ J. BELCHÍ \\ Universidad Autónoma de Madrid
}

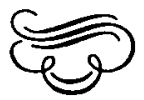

\section{Resumen}

Dos cuestiones parecen ser las que requieren mayor investigación actualmente en el contexto de las técnicas de potencial de aprendizaje: los efectos diferenciales, en distintos tipos de sujetos, del entrenamiento suministrado y la posibilidad de generalización del entrenamiento en el tiempo y a otras tareas.

Se presentan una serie de investigaciones llevadas a cabo con el objeto de analizar estas cuestiones en sujetos de 10 a 15 años con distintos niveles intelectuales; todas ellas se ban centrado en la técnica EPA: evaluación del potencial de aprendizaje, que utiliza material tipo Raven en sus entrenamientos.

Los resultados muestran la especificidad del entrenamiento suministrado aunque aparecen diferencias significativas en la generalización entre sujetos con $\dot{y}$ sin alteraciones orgánicas.

\section{Abstract}

Two issues seem to require at present more research in the context of the Techniques of Learning Potencial: The differential effects of the training provided in different types of subjects, and the possibility of extending the training in time as well as to other tasks.

Research studies carried out with the purpose of analyzing these matters in subjects between 10 and 15 with different intellectual level, are presented. All of them are based on the ALP techniques: Assessment of Learning Potential, using Raven type material in the training. Results show the specificity of the training provided, though significant differences appear in subjects with and without an organic etiology.

Dirección del primer autor: Universidad de Granada. Facultad de Filosofía y Letras. Departamento de Personalidad, Evaluación y Tratamiento. Campus universitario de Cartuja, 18011 Granada. 
Desde que distintos grupos de investigación empezaran a trabajar con el paradigma test-entrenamientó-test como alternativa a la evaluación tradicional de la inteligencia y comenzara a plasmarse a nivel empírico el concepto de potencial de aprendizaje hasta nuestros días, hemos acumulado gran cantidad de datos sobre el efecto que los tratamientos a corto plazo, que configuran estas técnicas, producen en los sujetos.

Se acepta hoy que, en general, en esta situación se producen en los sujetos aumentos significativos en las puntuaciones de las pruebas utilizadas como pre y post-test, y los interrogantes se dirigen hacia aspectos metodológicos relacionados con la validez de estas técnicas de evaluación.

Dos son, desde nuestro punto de vista, las cuestiones que demandan mayor investigación. Por un lado, la referida a los efectos diferenciales del entrenamiento en distintos grupos de sujetos: con o sin alteraciones orgánicas, con alto o bajo nivel intelectual, etc.

Por otro, referente a la posibilidad de generalización del entrenamiento suministrado, entendida ésta a los dos niveles posibles: como mantenimiento en el tiempo (estabilidad) de las mejoras obtenidas, y como transfer del aprendizaje a otros problemas más o menos parecidos.

Para nosotros, ambas cuestiones no tienen por qué darse por separado. Es posible que los efectos diferenciales del entrenamiento se manifiesten precisamente en la posibilidad de generalización de los efectos de éste en el tiempo o a otras tareas, tal y como afirmaron Pinillos (1981) y Campione et al. (1985) y como parecía desprenderse en los resultados obtenidos en algunos trabajos (Brown y Ferrara 1985; Fernández-Ballesteros, Campllonch y Macià, 1982, etc.).

Como hemos planteado en otras ocasiones (veáse Fernández-Ballesteros, Calero, Campllonch y Belchí, en prensa), estas técnicas requieren comprobar su fiabilidad (entendida ésta como mantenimiento de las ganancias obtenidas tras el entrenamiento) y su validez, tanto predictiva como de constructo o experimental (entendiéndose ésta como la especificidad y efectividad del entrenamiento suministrado).

Por otro lado, si pretendemos que sean instrumentos eficaces para la programación de tratamientos cognitivos a largo plazo, es necesario, tal y como han planteado numerosos autores (Brown y French, 1982; Sternberg, 1982; Lidz, 1983; Forns y Boada, 1985, etc.), que nos permitan la elaboración de un perfil cognitivo del sujeto, y esto requiere un análisis, desde los modelos de procesamiento de la información, no sólo de las tareas, sino también de las distintas posibilidades de transfer de la tarea entrenada.

Presentamos una serie de experimentos dirigidos a profundizar en esta compleja cuestión. Estos trabajos, que fueron realizados como parte de investigaciones más complejas sobre las técnicas de evaluación del potencial de aprendizaje (Belchí, 1985; Calero, 1986a; Calero, 1986b), suponen una prolongación del trabajo presentado en 1982 por Fernández-Ballesteros, Campllonch y Maciá, centrado en el entrenamiento sobre matrices progresivas (tipo Raven) que constituye el instrumento para la Evaluación del Potencial de Aprendizaje: EPA (Fernández-Ballesteros, Calero, Campllonch y Belchí, en prensa). En dicha investigación concluyeron que el entrenamiento suministrado no se generalizaba a tests que supuestamente requerían productos similares, tales como el TIG-1 y el subtest Espacial del PMA, y que la duración de las ganancias parecía estar relacionada con el nivel ba- 
sal de inteligencia de los sujetos, manteniéndose éstas en algunos grupos en períodos de más de un año.

En este caso nos centramos sobre los posibles efectos diferenciales del entrenamiento en distintos grupos de sujetos y en la comparación de la especificidad del mismo, intentando analizar las posibilidades del transfer en los términos planteados por Brown y French (1982), tal y como describiremos a continuación.

\section{PROCEDIMIENTO GENERAL Y MATERIAL DE ENTRENAMIENTO}

Se ha seguido en todos los casos un diseño pretest-entrenamiento-posttest.

En el pretest y posttest, se ha utilizado el test de Raven, escala General, Series A, Ab, B, C, D y E, en su modo de aplicación habitual.

$\mathrm{El}$ entrenamiento se ha efectuado en pequeño grupo, con una presentación en diapositivas de problemas similares a los de la prueba y un sistema de interacción altamente estructurado, tal y como se incluye en el instrumento para la Evaluación del Potencial de Aprendizaje EPA (véase, Fernández-Ballesteros, Calero, Campllonch y Belchí, en prensa).

En el primer experimento se han entrenado, fundamentalmente, las series $\mathrm{A}, \mathrm{Ab}$ y $\mathrm{B}$ del test de Raven, con un juego de 96 diapositivas, dos por problema, una de presentación, y otra con la solución correcta.

El entrenamiento de la Serie A está constituido por problemas de completación de un diseño. El de la serie $\mathrm{Ab}$ lo constituyen fundamentalmente problemas de simetria. El de las serie $B$ se basa en aplicación de razonamiento analógico a diseños en forma de matriz $2 \times 2$. También se entrena el concepto de seriación y su aplicación en los primeros problemas que constituyen la serie $C$.

En el segundo experimento, el material que constituye el entrenamiento está formado por 17 problemas seleccionados de entre los mencionados que entrenan las series A y B y 39 problemas que entrenan la serie C: problemas de seriación en un diseño $3 \times 3$ con ampliación de las figuras, disminución de las figuras, suma, resta y traslación; y la Serie E, problemas similares en los que se combinan varias de estas operaciones.

En el tercer experimento se seleccionan 29 problemas de matrices $3 \times 3$, 9 que entrenan problemas de la serie $C$ y 20 que entrenan la serie $E$.

Desde nuestro punto de vista, siguiendo la clasificación planteada por Brown y French (1982), la aplicación del entrenamiento a problemas de las series $\mathrm{A}, \mathrm{Ab}$ y $\mathrm{B}$, en el primer experimento, constituirian transfer cercano, dado que éstos exigen para su resolución las mismas reglas que han sido entrenadas. La resolución de las serie D exigiria trnasfer intermedio, puesto que estos problemas se resolverían con las reglas entrenadas, pero el diseño se amplía de matrices $2 \times 2$ a matrices $3 \times 3$. La serie $C$ en su mayoría y la Serie $\mathrm{E}$ exigirían tranfer distante, pues requieren reglas distintas de las entrenadas.

En el caso de los experimentos II y III las series entrenadas y, por tanto, que requieren transfer cercano son la $\mathrm{C}$ y la $\mathrm{E}$, y las restantes exigirían transfer distante. 


\section{EXPERIMENTO I}

\section{Objetivo}

Se trata de comprobar si aparecen diferencias significativas en la ganacia post-tratamiento, en el mantenimiento de la misma por un período de un año, y/o en la generalización del entrenamiento suministrado a las series no entrenadas ( $C, D$ y E) del test de Raven, escala general, en sujetos que presentan déficits intelectivos, relacionados con la presencia o no presencia de alteraciones orgánicas; relacionados con su puntuación inicial en el test de Raven y/o relacionados con la cuantía de su puntuación de ganancia (diferencia post-pretest).

\section{Procedimiento}

Se ha seguido el procedimiento antes descrito, aplicando un retest al año, en el que utilizamos de nuevo el Raven escala General.

\section{Muestra}

48 sujetos de 10 a 14 años de edad pertenecientes a un Centro de Educación Especial, 24 de ellos con alteraciones orgánicas y 24 en los cuales no se detectaron alteraciones orgánicas, en las distintas exploraciones practicadas. Nivel Intelectual entre 50 y 80 con media de 63.025 (según WISC).

\section{Análisis Estadístico}

ANOVA de las ganancias totales y de la puntuación ganancia de cada una de las series que componen las pruebas entre orgánicos/no orgánicos, altos/bajos en WISC inicial y ganadores/no ganadores en la EPA.

\section{Resultados}

Tal y como se refleja en la Tabla I, aparecen diferencias significativas entre orgánicos y no orgánicos en el incremento en las puntuaciones totales y en las series $\mathrm{B}$ y $\mathrm{D}$, y en el mantenimiento, después de un año, de estas ganancias. En relación con los análisis realizados entre altos y bajos, no aparecen diferencias significativas. Entre ganadores y no ganadores, aparecen diferencias significativas en las ganancias obtenidas por el primer grupo frente al segundo en todas las series de Raven y se mantienen las diferencias significativas en las series $\mathrm{B}, \mathrm{y} \mathrm{D}$, en el retest efectuado al año. 


\section{TABLA I}

Puntuaciones medias directas totales y de cada una de las series obtenidas por el conjunto de los sujetos divididos en: 1 . orgánicos y no orgánicos; 2 . altos y bajos según CI y

3. ganadores $y$ no ganadores

\begin{tabular}{lcc}
\hline \multicolumn{1}{c}{1} & Orgánicos & No orgánicos \\
\hline Raven Pre- & 16.5833 & 19.7917 \\
Raven Post- & 19.7919 & $27.000 * *$ \\
Post-Pre & 3.2083 & 7.2083 \\
Raven al año & 18.7010 & $25.8750 * *$ \\
Post-Pre al año & 2.1177 & $6.0833 * *$ \\
Serie A, pretest & 7.8333 & 8.375 \\
Serie A, post-pre & 0.3330 & 1.000 \\
Seire A, post-pre al año & 0.647 & 1.2500 \\
Serie B, pretest & 4.0830 & 4.9580 \\
Serie B, post-pre & 1.125 & 2.3300 \\
Serie B, post-pre al año & 0.2350 & 1.8120 \\
Serie C, pretest & 1.7500 & 2.5000 \\
Serie C, post-pre & 1.4166 & 1.2900 \\
Serie C, post-pre al año & 1.1170 & 1.3120 \\
Serie D, pretest & 2.0420 & 2.2080 \\
Serie D, post-pre & 0.4166 & $2.6250 * *$ \\
Serie D, post-pre al año & 0.2353 & $2.6870 * *$ \\
Serie E, pretest & 0.8750 & 1.2500 \\
Serie E, post-pre & 0.0416 & 0.2500 \\
Serie E, post-pre al año & 0.0000 & $-0.2500-$ \\
\hline
\end{tabular}

\begin{tabular}{lcc}
\hline \multicolumn{1}{c}{2} & Altos & Bajos \\
\hline Racen pre- & 23.8636 & $12.19000 * *$ \\
Raven post & 29.2727 & $17.4976 \% *$ \\
Post-Pre & 5.4091 & 5.3077 \\
Raven al año & 27.3636 & $16.2733 * *$ \\
Post-pre al año & 3.5000 & 4.0833 \\
Serie A, pretest & 9.7727 & \\
Serie A, post-pre & 0.4545 & 6.6900 \\
Serie A, post-pre al año & 0.0666 & 0.9230 \\
Serie B, pretest & 5.9090 & 1.3333 \\
Serie B, post-pre & 1.1818 & 3.3460 \\
Serie B, post-pre al año & 0.8666 & 1.7307 \\
Serie C, pretest & 3.6818 & 1.0550 \\
Serie C, post-pre, & 1.1360 & $0.8070 \% *$ \\
Serie C, post-pre al año & 1.2000 & 1.1538 \\
Serie D, pretest & 3.6818 & 1.2222 \\
Serie D, post-pre & 1.6360 & $0.8070 * *$ \\
Serie D, post-pre al año & 0.8000 & 1.4900 \\
Serie E, pretest & 1.4545 & 1.8800 \\
Serie E, post-pre & 0.3636 & 0.7300 \\
Serie E, post-pre al año & 0.0000 & -0.1500 \\
\hline
\end{tabular}


(Continuación)

\begin{tabular}{|c|c|c|}
\hline 3 & Ganadores & No ganadores \\
\hline $\begin{array}{l}\text { Raven Pre- } \\
\text { Raven-Post } \\
\text { Post-Pre } \\
\text { Raven al año } \\
\text { Post-Pre al año }\end{array}$ & $\begin{array}{r}19.0952 \\
29.6667 \\
10.8475 \\
27.0475 \\
7.9523\end{array}$ & $\begin{array}{r}17.4800 \\
18.5185 \approx * \\
1.0185 \approx * \\
18.5926 \approx * \\
1.1126 * *\end{array}$ \\
\hline $\begin{array}{l}\text { Serie A, pretest } \\
\text { Serie A, post-pre } \\
\text { Serie A, post-pre al año }\end{array}$ & $\begin{array}{l}8.3300 \\
1.3330 \\
1.0000\end{array}$ & $\begin{array}{l}7.9259 \\
0.1870 \\
0.6842\end{array}$ \\
\hline $\begin{array}{l}\text { Serie B, pretest } \\
\text { Serie B, post-pre } \\
\text { Serie B, post-pre al año }\end{array}$ & $\begin{array}{l}4.4760 \\
3.5380 \\
2.4286\end{array}$ & $\begin{array}{l}4.5550 \\
0.2922 \div \% \\
-0.0526 \approx\end{array}$ \\
\hline $\begin{array}{l}\text { Serie C, pretest } \\
\text { Serie C, post-pre } \\
\text { Serie C, post-pre al año }\end{array}$ & $\begin{array}{l}2.3890 \\
2.0428 \\
1.3120\end{array}$ & $\begin{array}{l}1.8518 \\
0.9200 \\
0.4736\end{array}$ \\
\hline $\begin{array}{l}\text { Serie D, pretest } \\
\text { Serie D, post-pre. } \\
\text { Serie D, post-pre al año }\end{array}$ & $\begin{array}{l}2.4760 \\
3.2857 \\
2.0714\end{array}$ & $\begin{array}{l}1.8518 \\
0.2190 * * \\
0.8947\end{array}$ \\
\hline $\begin{array}{l}\text { Serie E, pretest } \\
\text { Serie E, post pre } \\
\text { Serie E, post-pre al año }\end{array}$ & $\begin{array}{l}0.7140 \\
0.6570 \\
0.0000\end{array}$ & $\begin{array}{c}1.2960 \\
-0.084 \\
-0.4210\end{array}$ \\
\hline
\end{tabular}

* Anova significativo.

\section{EXPERIMENTO II}

\section{Objetivo}

Se trata de comprobar si sujetos de la misma edad, con un nivel intelectual normal, obtienen puntuaciones similares a las de los sujetos de más bajo nivel intelectual cuando se les entrena en una tarea que no conocen.

\section{Procedimiento}

Se sigue el general, pero utilizando en la fase de entrenamiento el material mencionado anteriormente.

\section{Muestra}

64 sujetos de 12 a 15 años, pertenecientes a un colegio público de zona rural. Divididos en dos grupos, control y experimental, y en tres niveles intelectuales (según Cattell-2): nivel Bajo (CI menor de 90) grupo experimental $=13$, grupo control $=11$; nivel intelectual Medio (CI entre $90 \mathrm{y}$ 110 ) g. experimental $=13$, g. control $=14$; nivel Alto, $\mathrm{CI}$ (mayor de 110) g. experimental $=7$, g. control $=6$. 


\section{Análisis Estadístico}

Estadístico $t$ de Student entre puntuaciones medias entre grupos Experimental y Control, para cada uno de los grupos intelectuales.

\section{Resultados Obtenidos}

Tal y como refleja la Tabla II, aparecen incrementos en las puntuaciones de las series entrenadas $\mathrm{C}$ y $\mathrm{E}$, aunque no se dan a un nivel de significación deseado. No aparecen aumentos en las puntuaciones de las demás series de la prueba.

Disminución de errores en 9 láminas de la serie $\mathrm{E}$ y en 6 de la serie $\mathrm{C}$.

\section{TABLA II}

Resultados obtenidos en el experimento número dos. Puntuaciones medias y desviaciones típicas en los grupos, experimental y control en las series entrenadas $(A, A b$ y $B)$ y en las series no entrenadas $(C, D$ y $E$ )

\begin{tabular}{|c|c|c|c|c|c|c|c|c|c|c|}
\hline & \multicolumn{5}{|c|}{ G. EXPERIMENTAL } & \multicolumn{5}{|c|}{ G. CONTROL } \\
\hline & \multicolumn{2}{|c|}{ Media } & \multirow[t]{2}{*}{ D } & \multicolumn{2}{|c|}{ D. típica } & \multicolumn{2}{|c|}{ Media } & \multirow[t]{2}{*}{ D } & \multicolumn{2}{|c|}{ D. típica } \\
\hline & Pre- & Post- & & Pre- & Post- & Pre- & Post- & & Pre- & Post- \\
\hline \multicolumn{11}{|c|}{ SERIES A-Ab-B } \\
\hline $\begin{array}{l}\text { N. Bajo } \\
\text { N. Medio } \\
\text { N. Alto }\end{array}$ & $\begin{array}{l}32,16 \\
33,54 \\
34,57\end{array}$ & $\begin{array}{l}33,16 \\
34,46 \\
34,14\end{array}$ & $\begin{array}{l}1 \\
, 92 \\
-, 43\end{array}$ & $\begin{array}{c}2,58 \\
2,54 \\
1,18\end{array}$ & $\begin{array}{l}2,79 \\
1,55 \\
1,64\end{array}$ & $\begin{array}{l}30,9 \\
32,75 \\
33,67\end{array}$ & $\begin{array}{l}32,8 \\
33,75 \\
35\end{array}$ & $\begin{array}{l}1,9 \\
1 \\
1,33\end{array}$ & $\begin{array}{l}2,88 \\
1,92 \\
1,11\end{array}$ & $\begin{array}{l}2,27 \\
1,83 \\
1\end{array}$ \\
\hline \multicolumn{11}{|c|}{ SERIES C-D-E } \\
\hline $\begin{array}{l}\text { N. Bajo } \\
\text { N. Medio } \\
\text { N. Alto }\end{array}$ & $\begin{array}{l}19,5 \\
20,85 \\
25,57\end{array}$ & $\begin{array}{l}22,58 \\
23,69 \\
26,85\end{array}$ & $\begin{array}{l}3,08 \\
2,84 \\
1,28\end{array}$ & $\begin{array}{l}4,25 \\
3,35 \\
3,2\end{array}$ & $\begin{array}{l}4,11 \\
4,56 \\
5,36\end{array}$ & $\begin{array}{l}17,7 \\
24,33 \\
21,33\end{array}$ & $\begin{array}{l}20,4 \\
21,67 \\
25,83\end{array}$ & $\begin{array}{r}2,7 \\
-2,7 \\
4,5\end{array}$ & $\begin{array}{l}4,58 \\
5,89 \\
4,11\end{array}$ & $\begin{array}{l}3,38 \\
4,11 \\
3,48\end{array}$ \\
\hline
\end{tabular}

VALORES DEL ESTADISTICO «t» de Student

\begin{tabular}{lccccccc}
\hline \multirow{2}{*}{ Grupo } & \multicolumn{2}{c}{ N. Bajo } & & \multicolumn{2}{c}{ N. Medio } & & \multicolumn{2}{c}{ N. Alto } \\
\cline { 2 - 3 } & Exper. & Control & Exper. & Control & & Exper. & Control \\
\hline Series A-Ab-B & 1.414 & 2.141 & 1.616 & 1.732 & & 1.162 & 2.000 \\
Series C-D-E & 3.031 & 2.613 & 2.945 & 2.245 & & 0,901 & 2.875 \\
\hline
\end{tabular}

\section{EXPERIMENTO III}

\section{Objetivo}

Comprobar la especificidad del entrenamiento seleccionando los problemas de mayor índice de dificultad.

\section{Procedimiento}

El material de entrenamiento en este caso estuvo constituido por 29 problemas $3 \times 3$ seleccionados de entre los utilizados en el entrenamiento an- 
terior porque habían mostrado su efectividad y porque la modalidad no incluía dibujos concretos. Nueve entrenaban la Serie $\mathrm{C}$, items números 1, 2, $3,4,5,6,8,9,11$ y 12 . Los veinte restantes entrenaban todos los problemas de la serie E.

\section{Muestra}

66 sujetos de 11 a 15 años, pertenecientes a un colegio público de medio rural, divididos en grupo experimental y grupo control y en tres grupos según nivel intelectual, medido por el Raven: Bajos (menos de 90), 7 en el grupo experimental y 6 en el grupo control; Medios (CI entre 90 y 110), 18 en el grupo experimental y 18 en el grupo control, y Altos (CI de más de 110). 9 en el grupo experimental y 8 en el grupo control.

\section{Análisis Estadístico}

$t$ de Student entre grupos control y experimental en cada una de las categorías establecidas según $\mathrm{CI}$.

\section{Resultados}

Tal y como se refleja en la Tabla III, se obtienen diferencias significativas en las series C y E. Mayores ganancias en la Serie $\mathrm{C}$ para el grupo bajo, y similares en los tres grupos en la serie E. No aparecen diferencias significativas en las series no entrenadas.

TABLA III

Resultados obtenidos en el tercer experimento. Medias y desviaciones típicas en cada una de las series del Raven para los grupos experimental y control y cada uno de los niveles según CI inicial.

\begin{tabular}{|c|c|c|c|c|c|c|c|c|c|c|}
\hline & \multicolumn{5}{|c|}{ G. EXPERIMENTAL } & \multicolumn{5}{|c|}{ G. CONTROL } \\
\hline & \multicolumn{2}{|c|}{ Media } & \multirow[t]{2}{*}{ D } & \multicolumn{2}{|c|}{ D. típica } & \multicolumn{2}{|c|}{ Media } & \multirow[t]{2}{*}{ D } & \multicolumn{2}{|c|}{ D. típica } \\
\hline & Pre- & Post- & & Pre- & Post- & Pre- & Post- & & Pre- & Post- \\
\hline SERIE A & & & & & & & & & & \\
\hline $\begin{array}{l}\text { N. Bajo } \\
\text { N. Medio } \\
\text { N. Alto }\end{array}$ & $\begin{array}{l}10,28 \\
11,11 \\
11,77\end{array}$ & $\begin{array}{l}10,85 \\
11,61 \\
11,88\end{array}$ & $\begin{array}{l}0,57 \\
0,50 \\
0,11\end{array}$ & $\begin{array}{l}1,38 \\
0,93 \\
0,41\end{array}$ & $\begin{array}{l}0,83 \\
0,59 \\
0,31\end{array}$ & $\begin{array}{r}9,83 \\
11,05 \\
11,22\end{array}$ & $\begin{array}{l}10,33 \\
11,27 \\
11,66\end{array}$ & $\begin{array}{l}0,47 \\
0,22 \\
0,44\end{array}$ & $\begin{array}{l}0,89 \\
0,97 \\
1,03\end{array}$ & $\begin{array}{l}0,94 \\
0,80 \\
0,47\end{array}$ \\
\hline SERIE B & & & & & & & & & & \\
\hline $\begin{array}{l}\text { N. Bajo } \\
\text { N. Medio } \\
\text { N. Alto }\end{array}$ & $\begin{array}{r}6,57 \\
10,16 \\
11,44\end{array}$ & $\begin{array}{r}9,71 \\
11,44 \\
11,88\end{array}$ & $\begin{array}{l}3,14 \\
1,28 \\
0,44\end{array}$ & $\begin{array}{l}2,19 \\
1,86 \\
0,83\end{array}$ & $\begin{array}{l}1,27 \\
1,01 \\
0,31\end{array}$ & $\begin{array}{r}7,83 \\
10,27 \\
10,88\end{array}$ & $\begin{array}{l}9,16 \\
11 \\
11\end{array}$ & $\begin{array}{l}1,33 \\
0,73 \\
0,12\end{array}$ & $\begin{array}{l}2,96 \\
1,14 \\
1,36\end{array}$ & $\begin{array}{l}3,48 \\
1,37 \\
1,56\end{array}$ \\
\hline
\end{tabular}

\section{SERIE C}

N. Bajo

N. Medio

N. Alto 
TABLA III

(Continuación)

\begin{tabular}{|c|c|c|c|c|c|c|c|c|c|c|}
\hline & \multicolumn{5}{|c|}{ G. EXPERIMENTAL } & \multicolumn{5}{|c|}{ G. CONTROL } \\
\hline & \multicolumn{2}{|c|}{ Media } & \multirow[t]{2}{*}{ D } & \multicolumn{2}{|c|}{ D. típica } & \multicolumn{2}{|c|}{ Media } & \multirow[t]{2}{*}{ D } & \multicolumn{2}{|c|}{ D. típica } \\
\hline & Pre- & Post- & & Pre- & Post- & Pre- & Post- & & Pre- & Post- \\
\hline SERIE D & & & & & & & & & & \\
\hline $\begin{array}{l}\text { N. Bajo } \\
\text { N. Medio } \\
\text { N. Alto }\end{array}$ & $\begin{array}{r}6,57 \\
9,38 \\
10,22\end{array}$ & $\begin{array}{r}9,14 \\
9,33 \\
10,22\end{array}$ & $\begin{array}{c}2,57 \\
-0,5 \\
0,00\end{array}$ & $\begin{array}{l}1,49 \\
1,2 \\
0,78\end{array}$ & $\begin{array}{l}1,8 \\
1,1 \\
0,41\end{array}$ & $\begin{array}{c}5,5 \\
9,5 \\
10,55\end{array}$ & $\begin{array}{r}6,33 \\
9,77 \\
10,11\end{array}$ & $\begin{array}{r}0,83 \\
0,27 \\
-0,44\end{array}$ & $\begin{array}{l}3,77 \\
1,6 \\
0,83\end{array}$ & $\begin{array}{l}3,39 \\
1,22 \\
1,09\end{array}$ \\
\hline \multicolumn{11}{|l|}{ SERIE E } \\
\hline $\begin{array}{l}\text { N.Bajo } \\
\text { N. Medio } \\
\text { N. Alto }\end{array}$ & $\begin{array}{l}2,48 \\
4,33 \\
8,33\end{array}$ & $\begin{array}{r}4,85 \\
7,05 \\
10,44\end{array}$ & $\begin{array}{l}2,57 \\
2,75 \\
2,11\end{array}$ & $\begin{array}{l}1,16 \\
1,76 \\
1,76\end{array}$ & $\begin{array}{l}1,45 \\
2,52 \\
1,57\end{array}$ & $\begin{array}{l}1,33 \\
3,83 \\
6,88\end{array}$ & $\begin{array}{l}2,00 \\
4,44 \\
7,88\end{array}$ & $\begin{array}{l}0,67 \\
0,61 \\
1,00\end{array}$ & $\begin{array}{l}1,1 \\
1,46 \\
2,18\end{array}$ & $\begin{array}{l}1,52 \\
2,08 \\
2,33\end{array}$ \\
\hline \multicolumn{11}{|l|}{ TOTAL } \\
\hline $\begin{array}{l}\text { N. Bajo } \\
\text { N. Medio } \\
\text { N. Alto }\end{array}$ & $\begin{array}{l}31,28 \\
43,94 \\
50,55\end{array}$ & $\begin{array}{l}43,57 \\
50,22 \\
55,11\end{array}$ & $\begin{array}{r}12,29 \\
6,28 \\
4,56\end{array}$ & $\begin{array}{l}4,86 \\
4,16 \\
4,94\end{array}$ & $\begin{array}{l}5,01 \\
4,32 \\
3,28\end{array}$ & $\begin{array}{l}30,16 \\
43,38 \\
49,66\end{array}$ & $\begin{array}{l}33,16 \\
45,22 \\
50,88\end{array}$ & $\begin{array}{l}3,00 \\
1,84 \\
1,22\end{array}$ & $\begin{array}{r}10,89 \\
3,26 \\
3,74\end{array}$ & $\begin{array}{r}11,2 \\
3,5 \\
4,3\end{array}$ \\
\hline
\end{tabular}

VALORES DEL ESTADISTICO «t» de Student

\begin{tabular}{lrrrrrrr} 
& \multirow{2}{*}{ Nivel } & \multicolumn{2}{c}{ N. Bajo } & \multicolumn{2}{c}{ N. Medio } & & \multicolumn{2}{c}{ N. Alto } \\
\cline { 2 - 3 } \cline { 6 - 7 } & G. Exp. & G. Ctrol. & G. Exp. & G. Ctrol. & G. Exp. & G. Ctrol. \\
\hline Serie A & 1.549 & 2.236 & 1.843 & 0.990 & & 0.554 & 1.315 \\
Serie B & 3.791 & 2.696 & 2.997 & 2.179 & & 1.315 & 0.262 \\
Serie C & 5.605 & -0.466 & 4.189 & 0.000 & 3.499 & 0.262 \\
Serie D & 4.203 & 1.051 & -0.187 & 0.892 & & 0.000 & -1.835 \\
Serie E & 3.422 & 1.195 & 5.030 & 1.313 & 3.919 & 2.121 \\
\hline
\end{tabular}

\section{DISCUSION}

Vamos a centrarnos en primer lugar sobre los posibles efectos diferenciales del entrenamiento con la EPA, en distintas muestras de sujetos.

En los trabajos realizados anteriormente con esta técnica de entrenamiento (Campllonch y Fernández-Ballesteros, 1981; Fernández-Ballesteros, Campllonch y Macià, 1982) se había puesto de manifiesto que los efectos del mismo se centraban en las series A, Ab y B. En los experimentos que hemos realizado se vuelve a producir el mismo efecto, tanto en sujetos con CI normal como en sujetos con déficits intelectivos. Este efecto positivo se da también en sujetos con alteraciones orgánicas.

Entre sujetos orgánicos y no orgánicos, aparecen diferencias significativas en el incremento de las puntuaciones en la serie $\mathrm{D}$, que como hemos apuntado no se entrena directamente, sino que se puede señalar como de transfer intermedio. Esas diferencias de puntuaciones obtenidas tras el entrenamiento se mantienen después de un año (Tabla I).

Sin embargo, en los distintos retest realizados no aparecen diferencias 
en el mantenimiento de puntuaciones de ganancia en las series entrenadas entre unos grupos y otros.

En relación con el nivel intelectual no aparecen, en los distintos experimentos realizados, diferencias significativas en ninguna de las series entrenadas, ni en el mantenimiento de las ganancias véanse las (Tablas I, II y III). Pues, aunque en el último experimento (Tabla III) aparecen mayores ganancias en la serie $C$ para el Grupo Bajo, estas diferencias son explicables si tenemos en cuenta que en los otros grupos es difícil que el entrenamiento tenga algún efecto, ya que la puntuación de partida de los sujetos está próxima al techo de la serie (12 puntos).

Estos resultados van en contra de los planteamientos hechos por Haywood (1977), Budoff (1973) y Campllonch y Fernández-Ballesteros (1981), que suponían que los sujetos de menor nivel intelectual conseguirían mejoras mayores tras el entrenamiento. Debemos que tener en cuenta, no obstante, que no hemos trabajado con un grupo extremo de nivel intelectual como lo hicieron estos últimos autores.

En relación con la generalización del entrenamiento, que era la segunda cuestión que nos habíamos planteado, parece claro que el entrenamiento suministrado por medio de esta técnica es bastante específico y las posibilidades de transfer reflejadas por todos los grupos de sujetos han sido en tareas cercanas, mejorando la resolución en problemas similares a los entrenados, o intermedias, mejorando la serie $\mathrm{D}$ a partir del entrenamiento en la serie $\mathrm{B}$, en sujetos sin alteraciones orgánicas.

No se ha obtenido transfer distante, que es el que exige, desde nuestro punto de vista, aplicar el entrenamiento de las primeras series más simples a las más complejas, o el de estas series a la serie D (experimento III) aunque los problemas entrenados sean de mayor dificultad que los probados $\mathrm{y}$ los sujetos posean un nivel intelectual normal o alto.

Estos resultados se ponen de manifiesto también cuando comparamos a los sujetos ganadores y no ganadores en la EPA, pues, si bien al principio aparecen diferencias significativas en todas las series del Raven, en el retest realizado al año las diferencias entre estos grupos se centran en las series $B$ y $\mathrm{D}$, o sea, en los ítems entrenados y en las tareas que exigen transfer intermedio.

\section{CONCLUSIONES}

Del examen de los resultados podemos concluir que el entrenamiento sobre el Raven que se realiza con la técnica EPA es efectivo en las series A, $\mathrm{Ab}$ y $\mathrm{B}$ de la prueba Raven, y se generaliza a la $\mathrm{D}$ en el caso de sujetos no orgánicos y en el caso de sujetos denominados ganadores (puntuación de ganancia de 5 ó más puntos). Y que las ganancias obtenidas tras el entrenamiento se mantienen en el tiempo en todos los grupos de sujetos.

No existen, pues, diferencias significativas ni en generalización del entrenamiento a otras tareas ni mantenimiento de las ganancias en el tiempo entre diferentes niveles intelectuales.

En relación con la especificidad del entrenamiento suministrado, los resultados obtenidos confirman las afirmaciones hechas por distintos autores (Campione et al., 1985; Brown y Ferrara, 1985; Chantal, 1984; Sternberg, 
1981) sobre la necesidad de que se den similitudes físicas entre contextos de las tareas para favorecer la transferencia, que determina, en cierto modo, el que ésta solo se dé en la medida en que se parta de elementos idénticos, confirmando así mismo los resultados obtenidos por Fernández-Ballesteros, Campllonch y Macià (1982).

Estos resultados son positivos como pruebas de fiabilidad y validez de esta técnica en el sentido apuntado al principio de este trabajo. Según se desprende de algunos trabajos realizados sobre generalización (Bray, Goodman y Justine, 1982; Campione et al., 1985; etc.), la comprensión por parte del sujeto de las demandas de la tarea es fundamental para conseguir la modificación de la estrategia entrenada, y en consecuencia la generalización; y en nuestra opinión, con la técnica EPA se da esta generalización, a los dos niveles al principio planteados, en la medida en que es posible dada la brevedad del entrenamiento suministrado.

En relación con la posibilidad de establecimiento de perfiles de generalización para los sujetos, pensamos que hay que seguir trabajando en este sentido, teniendo en cuenta que a partir de un entrenamiento tan corto es bastante difícil conseguir que ésta se dé a tareas distantes. Desde nuestro punto de vista, ésta será posible cuando las tareas impliquen los mismos procesos, no los mismos productos, y los saltos en complejidad, modalidad, contenido, etc., se vayan estableciendo según un sistema de aproximaciones sucesivas. Es importante, no obstante, establecer qué condiciones la facilitan y aplicarlo a estas técnicas de evaluación.

\section{Referencias}

BeLCHI, J. (1985). Potencial de Aprendizaje: Una crítica. Tesis de Licenciatura inédita. Universidad Autónoma de Madrid.

BraY, N. M.; GOODMAN, M. A. y JUSTINE, E. M. (1982). Task instruction and strategy transfer in the Directed forgetting performance of mentally retarded adolescents. Intelligence, $6(2), 187-200$.

BROWN, A. L. y CAMPIONE, J. C. (1982). Modifying intelligence or modifying cognitive skills: More than a semantic quibble? En D. K. Detterman y R. J. Sternberg (comps.): How an how much can intelligence be increased. Nueva Jersey: Ablex P. C., 215-230.

BROWN, A. L. y FERRARA, R. A. (1985). Diagnosis zones of proximal development. En J. Wertsch (comp.): Culture, communitation and cognition: Vygotskian perspectives. Cambridge: Cambridge U. P. 273-305.

BROWN, A. L. y FRENCH, L. A. (1979). The zone of potential development: Implications for intelligence testing in the year 2000. Intelligence, 3 (3), 255-273.

BuDOFF, M. (1970). Learning Potential: Assessing ability to reason in the educable mentally retarded. Acta Paedopsichiatrica (37), 293-309.

Calero, M. D. (1986a). Un estudio de un test de Potencial de Aprendizaje. Tesis Doctoral. Microficha. Servicio de Publicaciones Universidad de Granada.

CALERO, M. D. (1986b). La Generalización de los entrenamientos cognitivos. Comunicación presentada en el V Congreso Nacional de la Sociedad Española de Neuropsiquiatría Infanto-juvenil. Málaga.

CAMPIONE, J. C.; BROWN, A. L.; FerRara, R. A.; JONES, R. S. y Steinberg, E. (1985). Breakdowns in flexible use of information intelligence related differences in transfer following equivalents learning performance. Intelligence, 9 (4), 297-315.

Campllonch, J. M. y Fernández-Ballesteros, R. (1981). La Evaluación del Potencial de Aprendizaje. En R. Fernández-Ballesteros (comp.): Nuevas Aportaciones en Evaluación Conductual. Valencia: Alphaplus, 257-277.

Chantal, G. (1984). Transfer de connaissances dans la resolution de deux problèmes analogues. Archives du Psychologie, 52 (201), 121-132.

Díaz Fernández, O. (1982). Evaluación del Potencial de Aprendizaje. Tesis Doctoral inédita. Universidad de Santiago. 
Fernández-Ballesteros, R.; Calero, M. D.; Campllonch, J. M. y Belchí, J. (en prensa). Instrumento para la Evaluación del Potencial de Aprendizaje: EPA (versión experimental). Madrid: MEPSA.

FernánDEZ-BAllesteros, R.; CAMPLlONCH, J. M. y MACí́, A. (1982). Generalización del entrenamiento de estrategias cognitivas. En R. Fernández-Ballesteros (comp.): Evaluación de contextos. Universidad de Murcia: Servicio de Publicaciones, 259-276.

FORNS, M. y BOADA, H. (1985). Consideraciones sobre la zona de desarrollo Potencial desde la Evaluación Psicológica. Anuario de Psicologia, (33).

HAYwOOD, H. C. (1977). Alternatives to normative assessment. En P. Mittler, (comp.): Research to practice in mental retardation, II. Baltimore: U. Park Press.

LIDZ, C. S. (1983). Dinamic assessment and the preschool children. Comunicación presentada en la AAMD N. Convention. Boston: Massachusetts.

PinILlos, J. L. (1981). La mejora cientifica de la inteligencia. Análisis y Modificación de Conducta, 7 (14-15), 115-124.

SternberG, R. J. (1981). Testing and cognitive psychology. American Psichology (36), 1181-1189. 